\title{
A Modified Model to Estimate Single Peak Flow Stress Curves of Ti-IF Steel
}

\author{
Ehsan SHAFIEI and Ramin EBRAHIMI \\ Department of Materials Science and Engineering, School of Engineering, Shiraz University, Shiraz, Iran. \\ (Received on August 25, 2011; accepted on December 1, 2011)
}

\begin{abstract}
In this study a modified model, using a non linear estimation of strain hardening rate vs. strain up to the critical stress, has been developed to predict single peak dynamic recrystallization (DRX) flow stress curves. The critical stress and strain for initiation of dynamic recrystallization were determined using: (1) the strain hardening rate versus stress curve, (2) the constitutive equation. In order to evaluate the accuracy of the compound model, the mean error was calculated. The results indicate that the proposed model give a good estimate of the flow stress curves.
\end{abstract}

KEY WORDS: mathematical modeling; flow stress curve; hot deformation; Ti-IF steel.

\section{Introduction}

Deformation of metals and alloys at temperatures above $0.5 \mathrm{~T}_{\mathrm{m}}$ is a complex process in which mechanical working interacts with various metallurgical processes such as dynamic restoration including recovery and recrystallization, and phase transformation for polymorphous materials. ${ }^{1)}$ The understanding of these processes, however, enables the behavior of the metals and alloys. It is accepted by many authors that metals and alloys which have relatively low values of stacking fault energy (SFE) could recrystallize dynamically, whereas those of high SFE including bcc metals and alloys which behave in a manner similar to fcc metals of high SFE recovered dynamically only during the high temperature deformation. ${ }^{1,2)}$

The analysis of metal forming process such as hot rolling, forging and extrusion has been dependent on various parameters including constitutive relation which contributes to stress-strain curves at high temperatures, shape of workpiece and product, shapes of tools, friction, temperature, forming speed, etc. In such parameters, the constitutive equation is one of the most important factors having an effect on solution accuracy. ${ }^{3)}$

A number of research groups have attempted to develop constitutive equations of materials and suggested their own formulations by putting the experimentally measured data into one single equation. Misaka ${ }^{4)}$ and Shida ${ }^{5)}$ proposed the models giving the stress of carbon steels as a function of the strain, strain rate, temperature and carbon content. Shida's model takes account of the flow stress behavior of the steels in austenite, ferrite and in the two-phase regions. Voce suggested an approximate equation of stress-strain curve considering the dynamic recrystallization. Ebrahimi et al. obtained a mathematical model according to the phenomenological representation of the shape of the stress-strain curves that consists an additional constant. ${ }^{6-8)}$ Finally shafiei and Ebrahimi ${ }^{9)}$ proposed a new constitutive equation, using the extrapolation of dynamic recovery flow stress curves and kinetic equation for dynamic recrystallization (DRX) which did not consider the dynamic recovery and work hardening region before critical strain. Due to the importance of flow stress estimation of metals and alloys at high temperatures, the required forces for the deformation processes, dimensional accuracy of final products and simulation of processes; it is necessary to provide a model to eliminate the limitations of previous models to some extent.

With regards to the importance of macroscopic data from mechanical tests as compared to microscopic ones from metallurgical investigation, due to its less time and cost consuming nature, mathematical and macroscopic aspects of dynamic recrystallization process is considered in this model. Finally, a modified mathematical model was obtained to express the flow behavior of metals and alloys at high temperatures up to the critical strain which can be used as a complement for Shafiei and Ebrahimi's previous DRX model. This analysis has been done for the stress-strain curves under hot working condition for Ti-IF steel. However, this model is not dependent on the type of material and can be extended for any condition in which a single peak dynamic recrystallization occurs.

\section{Flow Curves}

As shown in Fig. 1, according to microstructural evolutions, material response can be principally divided into two categories during the hot deformation: dynamic recovery (DRV) type and DRX type.

For metals with DRV, the flow stress curves increase with strain in the initial deformation and reach constant in consequence of attaining the balance between work hardening and DRV (saturation stress, $\sigma_{\mathrm{s}}$ ). For metals with DRX, initially the flow stress increases with strain which is being 


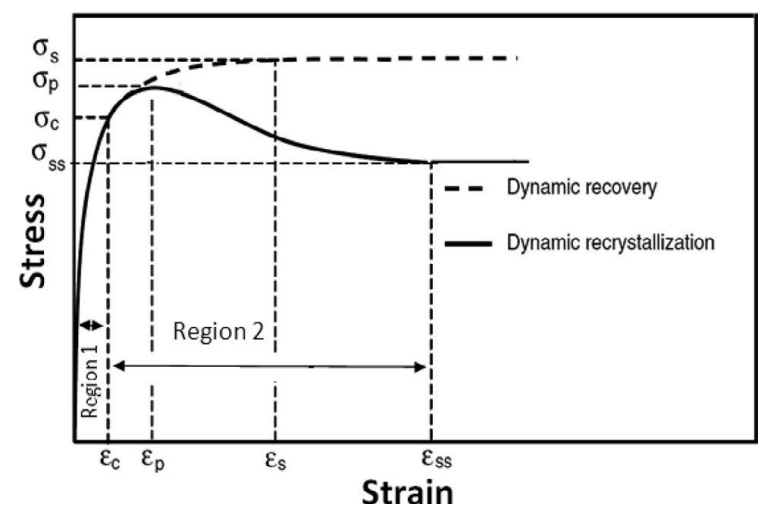

Fig. 1. Schematic representing of single peak flow stress curve at high temperature.

dominated by work hardening, and as DRX takes place upon critical strain $\left(\varepsilon_{\mathrm{c}}\right)$, the flow stress begins to decrease after reaching certain peak value. When the equilibrium is reached between softening due to DRX and work hardening, the curves drop to a steady state region $\left(\sigma_{\mathrm{ss}}\right)^{1,6)}$

\section{Strain Hardening Rate vs. Stress}

The change in the slope $(\theta=\mathrm{d} \sigma / \mathrm{d} \varepsilon)$ of the stress-strain curve with stress can be a good indication of the microstructural changes taking place in material. ${ }^{2)}$ All of the $\theta-\sigma$ curves for a particular alloy originate from a common intercept $\theta_{0}$ at $\sigma=0$. As shown in Fig. 2, the $\theta-\sigma$ curves have three segments, two of them are linear. The first linear segment decreases with stress for initial strain to the point where subgrains begin to form with a lower rate of increase in DRV. The curve gradually changes to the lower slope of the second linear segment up to the point where the critical stress $\sigma_{\mathrm{c}}$ is attained for initiating the dynamic recrystallization. The curve then drops off rather sharply to $\theta=0$ at peak stress. Extrapolation of the second linear segment of the $\theta$ $\sigma$ curve intercepts the $\sigma$ axis at the saturation stress. This would be the shape of the $\theta$ - $\sigma$ curve if dynamic recrystallization were absent with only dynamic recovery as the restorative mechanism operation. ${ }^{2,10,11)}$

\section{Initiation of Dynamic Recrystallzation}

Several attempts have been made to predict the initiation of DRX. For example, Ryan and McQueen observed that the presence of a stress peak at a constant strain rate flow curve leads to an inflection in plots of strain hardening rate, $\theta$, versus stress, $\sigma{ }^{9)}$ Moreover, the points of inflection in $\theta-\sigma$ plots where the experimental curves separate from the extrapolated lower linear segments give critical conditions for initiation of DRX. Later, Poliak and Jonas ${ }^{10)}$ have shown that this inflection point corresponds to the appearance of an additional thermodynamic degree of freedom in the system due to the initiation of DRX.

Furthermore, the method of Poliak and Jonas was subsequently simplified by Najafizadeh and Jonas. ${ }^{12)}$ In their approach, a third order polynomial equation was fitted to the $\theta-\sigma$ curves.

Several constitutive equations are available to model the flow curves at hot working conditions. Among them, the

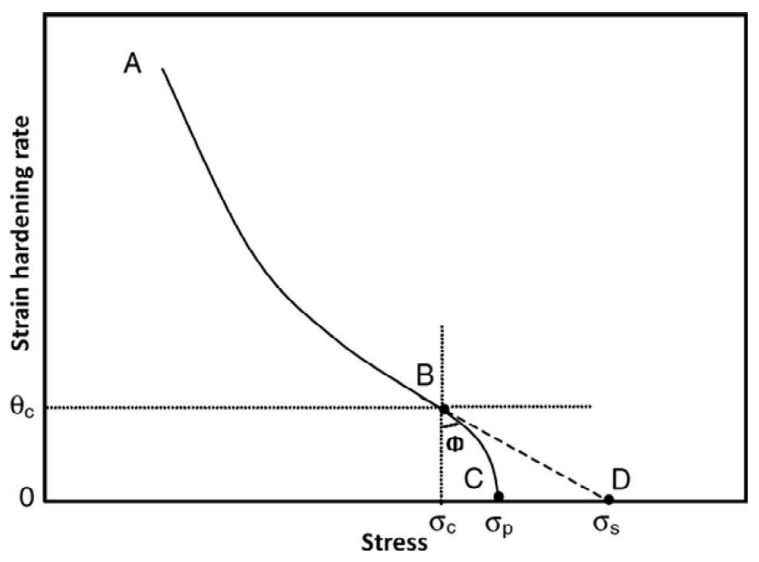

Fig. 2. Changes in the slope of the stress-strain curve with stress.

Cingara and McQueen equation ${ }^{6,9,13)}$ is of special importance. It deals with flow curves up to peak point which is the region of interest for determination of critical conditions for initiation of DRX. The application of this method on determining the critical strain has been previously analyzed on $\mathrm{Nb}$ steel by Ebrahimi and Solhjoo. ${ }^{14)}$

In this work, the critical stress and strain for initiation of dynamic recrystallization (DRX) were determined using: (1) the strain hardening rate versus stress curve, (2) the constitutive equation.

\subsection{Determination of Critical Stress}

The simple method of Najafizadeh and Jonas ${ }^{12)}$ was used for determination of the critical stress for initiation of DRX. The inflection point is detected by fitting a third order polynomial to the $\theta-\sigma$ curves up to the peak point as follows:

$$
\theta=A \sigma^{3}+B \sigma^{2}+C \sigma+D
$$

where $A, B, C$, and $D$ are constants for a given set of deformation conditions. The second derivative of this equation with respect to $\sigma$ can be expressed as:

$$
\frac{d^{2} \theta}{d \sigma^{2}}=6 A \sigma+2 B
$$

At critical stress for initiation of DRX, the second derivative becomes zero. Therefore,

$$
6 A \sigma+2 B=0 \rightarrow \sigma_{c}=-\frac{2 B}{6 A}
$$

\subsection{Determination of Critical Strain}

The flow stress up to the peak stress was modeled using the Cingara equation ${ }^{6,9)}$ as shown below:

$$
=\sigma_{p}\left[\left(\frac{\varepsilon}{\varepsilon_{p}}\right) \exp \left(1-\left(\frac{\varepsilon}{\varepsilon_{p}}\right)\right)\right]^{C}
$$

Where constant $C$ is an additional parameter to make the results acceptable and is obtained from logarithmic form of Eq. (4). The derivative of the true stress with respect to true strain yields the work hardening rate, $\theta$. Therefore, the $\theta$ formula using Eq. (4) may be expressed as:

$$
\theta=C \sigma\left(\frac{1}{\varepsilon}-\frac{1}{\varepsilon_{p}}\right)
$$


Table 1. Chemical composition of hot torsion specimens ( $\% \mathrm{Wt})$.

\begin{tabular}{ccccccccccccccc}
\hline Specimen & $\% \mathrm{C}$ & $\% \mathrm{Si}$ & $\% \mathrm{Mn}$ & $\% \mathrm{P}$ & $\% \mathrm{~S}$ & $\% \mathrm{~N}$ & $\% \mathrm{Al}$ & $\% \mathrm{Cr}$ & $\% \mathrm{Cu}$ & $\% \mathrm{Mo}$ & $\% \mathrm{Ni}$ & $\% \mathrm{Nb}$ & $\% \mathrm{~V}$ & $\% \mathrm{Ti}$ \\
\hline Ti-IF steel & 0.002 & 0.02 & 0.13 & 0.005 & 0.005 & 0.003 & 0.03 & 0.037 & 0.014 & 0.008 & 0.025 & 0.002 & 0.002 & 0.062 \\
\hline
\end{tabular}

In order to determine the critical strain, the second derivative of $\theta$ with respect to $\sigma$ must be zero. By solving this equation, the critical strain as a function of peak strain will obtain as follows:

$$
\frac{\varepsilon_{c}}{\varepsilon_{p}}=\frac{\sqrt{1-C}-(1-C)}{C}
$$

\section{Mathematical Modeling of Flow Stress Curves}

As shown in Fig. 1 the stress-strain curve represents two distinct regions. In region (1), the deformation of metals at elevated temperature shows work hardening plus dynamic recovery to a critical stress followed by region (2) which represents a single peak dynamic recrystallization flow stress curve.

\subsection{Mathematical Modeling of Single Peak DRX Flow Stress Curves, Region 2}

Using the extrapolation of DRV flow stress curves and kinetic equation for DRX, Shafiei and Ebrahimi ${ }^{9)}$ proposed the following equation for modeling single peak DRX flow curves for $\mathcal{E}_{c} \leq \varepsilon<\mathcal{E}_{s s}$ :

$$
\sigma=\sigma_{s}-\left(\sigma_{s}-\sigma_{c}\right) \exp \left(\dot{c}\left(\varepsilon_{c}-\varepsilon\right)\right)-\left(\sigma_{s}-\sigma_{s s}\right) X \ldots
$$

Where $C^{\prime}$ is a constant with metallurgical sense. According to the geometrical relations shown in Fig. 2, the value of $C^{\prime}$ can be formulized as Eqs. (8) and (9). $\sigma_{\mathrm{c}}, \sigma_{\mathrm{p}}, \sigma_{\mathrm{ss}}, \sigma_{\mathrm{s}}, \varepsilon_{\mathrm{c}}, X$ are critical stress, peak stress, DRX steady state stress, DRV saturation stress, critical strain and volume fraction of DRX, respectively.

$$
\begin{array}{r}
\tan \varphi=\frac{1}{\dot{c}} \ldots \ldots \ldots \ldots \ldots \ldots \\
\varphi=\frac{\pi}{2}+\operatorname{Arctan}\left(\frac{\sigma_{p} \varepsilon_{p}}{\left(\varepsilon_{c}-\varepsilon_{p}\right) \varepsilon_{c}}\right)
\end{array}
$$

\subsection{Mathematical Modeling of WH+DRV Flow Stress Curves, Region 1}

The derivative of the flow curve is positive up to the peak stress and then phenomenologically the slope of the stressstrain curve is negative and approaches zero to the steady state stress. The variations of the slope can be expressed by the following equation up to the critical strain:

$$
\theta=\frac{d \sigma}{d \varepsilon}=\frac{k}{\varepsilon}
$$

Where $k$ is an additional parameter in order to make the result of the model acceptable and must be determined for each condition. Solution of differential Eq. (10) with respect to $\varepsilon$, using boundary condition $\sigma=\sigma_{\mathrm{c}}$ at $\varepsilon=\mathcal{E}_{\mathrm{c}}$ is:

$$
\sigma=k \ln \frac{\varepsilon}{\varepsilon_{c}}+\sigma_{c} \quad \varepsilon_{0}<\varepsilon \leq \varepsilon_{c}, \varepsilon_{0} \neq 0
$$

Determination of $k$ can be simply done by fitting a logarithmic curve vs. experimentally measured data. The results

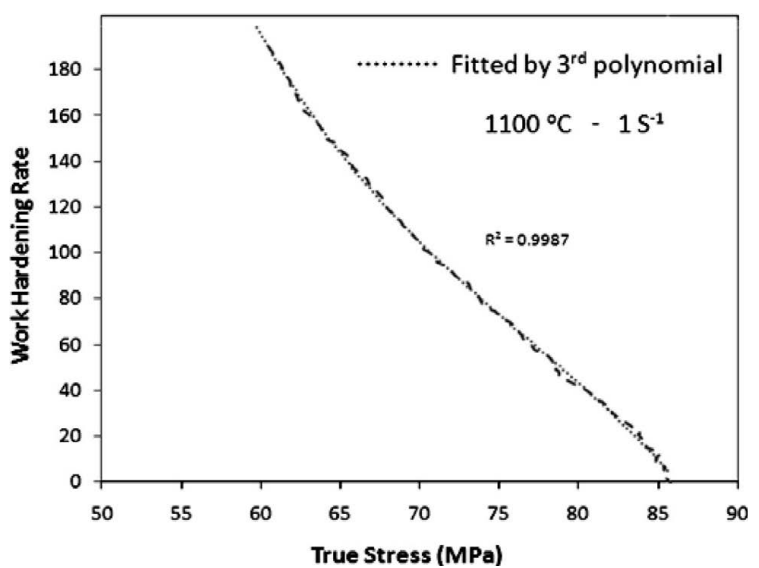

Fig. 3. The $\theta-\sigma$ curve ant it's corresponding third order polynomial.

show that $k$-value is a function of temperature and can be calculated by following relationship:

$$
k=m T+n .
$$

where $T$ is the absolute temperature and $m$ and $n$ are constant. Using a plot of $k$ vs. $T$, constants $m$ and $n$ can be determined. It is also possible to assume $k$ as a constant, but it shows a slight deviation from the experiments.

\section{Results and Discussion}

According to the experimental results of Ti-IF steel in the literature, ${ }^{15)}$ acquired from hot torsion test, all flow curves display a rapid initial increase to a maximum stress, characterized by a peak strain and peak stress, followed by a gradual fall to a steady stress which indicates a typical shape of DRX flow stress curves. Chemical composition of hot torsion specimens are presented in Table $\mathbf{1}$.

An example of $\theta-\sigma$ curves and its corresponding third order polynomial are shown in Fig. 3. As can be seen, the $3^{\text {rd }}$ curve has a good fit as previously demonstrated by Najafizadeh and Jonas. Therefore, this method was used to determine the value of critical stress at different deformation conditions. Using the flow curves, the values of critical strain were determined.

According to Najafizadeh and Jonas method, the present investigation indicates that the DRX starts when the normalized critical strain reaches to the value of about 0.43 which was obtained using the average values of normalized stress at different deformation temperature for Ti-IF steel. This deviates from the previous studies on steels which has been reported a value in the range of $0.6-0.8$. It should be noted that for some steel grades such as AK steel and 17-7 PH steel, the value of 0.52 and 0.46 have also been reported. ${ }^{13)}$

According to Fig. 3, as an example the normalized critical stress and strain at temperature of $1100^{\circ} \mathrm{C}$ and strain rate of $1 \mathrm{~s}^{-1}$ could be presented as: 


$$
\begin{gathered}
\frac{\sigma_{c}}{\sigma_{p}}=0.85 \\
\frac{\varepsilon_{c}}{\varepsilon_{p}}=0.431
\end{gathered}
$$

Also the plot of $\ln \left(\sigma / \sigma_{\mathrm{p}}\right)$ versus $1-\left(\varepsilon / \varepsilon_{\mathrm{p}}\right)+\ln \left(\varepsilon / \varepsilon_{\mathrm{p}}\right)$ can be used to determine the value of the constant $\mathrm{C}$. An example is shown in Fig. 4 for Ti-IF steel at temperature of $1100^{\circ} \mathrm{C}$ and strain rate of $1 \mathrm{~s}^{-1}$. The flow curve modeling was performed for each deformation condition. By substituting the value of $\mathrm{C}=0.401$ in Eq. (6), the normalized critical strain can be determined as:

$$
\frac{\varepsilon_{c}}{\varepsilon_{p}}=0.436
$$

According to this method, using the average value of $\mathrm{C}$, the normalized critical strain was obtained as 0.429 which is in a good agreement with the result of Najafizadeh and Jonas method.

The good agreement between the results of Cingara ${ }^{6,9,13)}$ equation and work hardening rate analysis in the case of present investigation is interesting. However, the constitutive equations such as the Cingara equation have several limitations. Many research results on hot working of steels reported a value in the range of $0.6-0.8$ for normalized critical strain. ${ }^{11,13)}$ This implies that the value of $\mathrm{C}$ should be between -1.25 and -15 respectively. However, the negative value of $\mathrm{C}$ is impossible.

In order to evaluate the temperature dependence of $k$-value, the plot of $k$-value vs. temperature was used. Also, using the linear trend line of the plot; $k$-value vs. T made it possible to determine the values of $m$ and $n$. It is important to mention that based on the existing flow curves, the value of $k$ were obtained as a temperature sensitive parameter. Therefore, to evaluate the strain rate dependency of this parameter, it is necessary to obtain the plot of $k$ vs. strain rate.

Figure 5 shows the relationship between $k$-value and $T$ and from this, Eq. (12) changes into:

$$
k=-0.077 T+119.2
$$

This enables to estimate $k$-value at any other temperature with a reasonable accuracy.

Figure 6 shows the calculated flow stress by Cingara ${ }^{6}$ and presented model for $\mathrm{WH}+\mathrm{DRV}$ region up to the critical

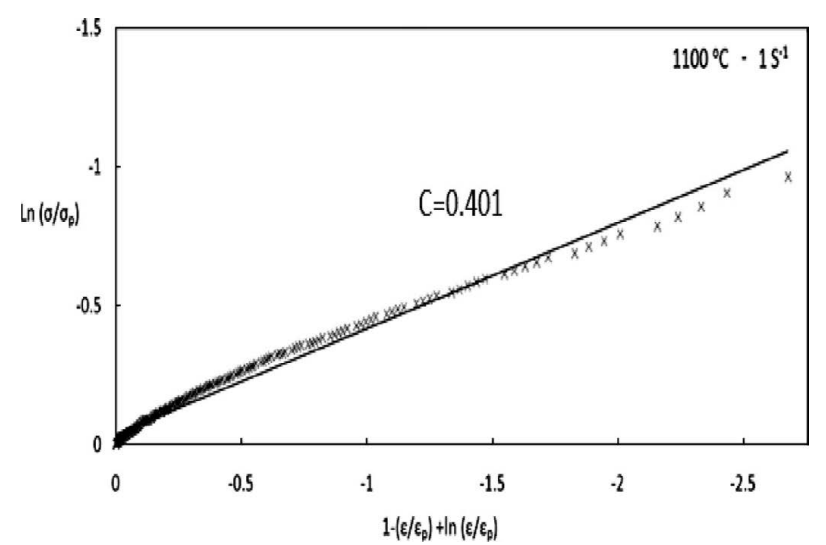

Fig. 4. Plot for calculation of Cingara constant. strain. The results indicate that the Cingara equation does not have a good capability to estimate the flow stress at critical strain for the alloy of this study. Therefore, in contrast to Cingara equation, the presented model may have more capable to predict flow stress curves up to the critical stress. Also, it should be considered that another limit of Cingara is its disability of prediction of flow stress at very low strains that the work hardening rate has very high values.

Using DRV+WH model as a complement for presented DRX model, made it possible to predict flow stress curves before and after critical strain. As shown in Fig. 7, the stress-strain curves predicted by using presented compound model are in a good agreement with experimentally mea-

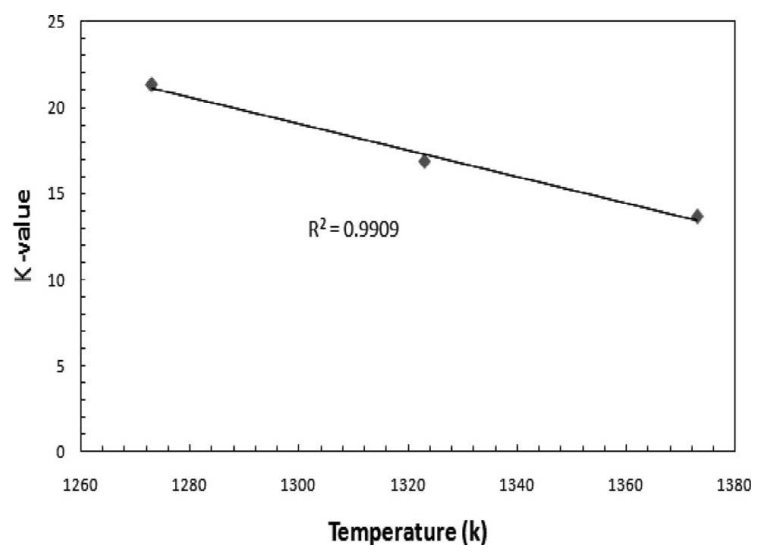

Fig. 5. Variations of k-value with temperature.

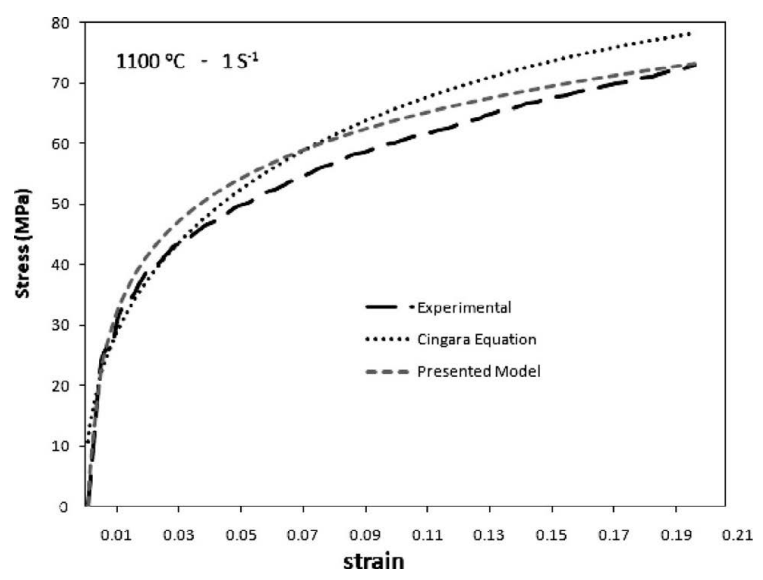

Fig. 6. Comparison of presented model and Cingara equation.

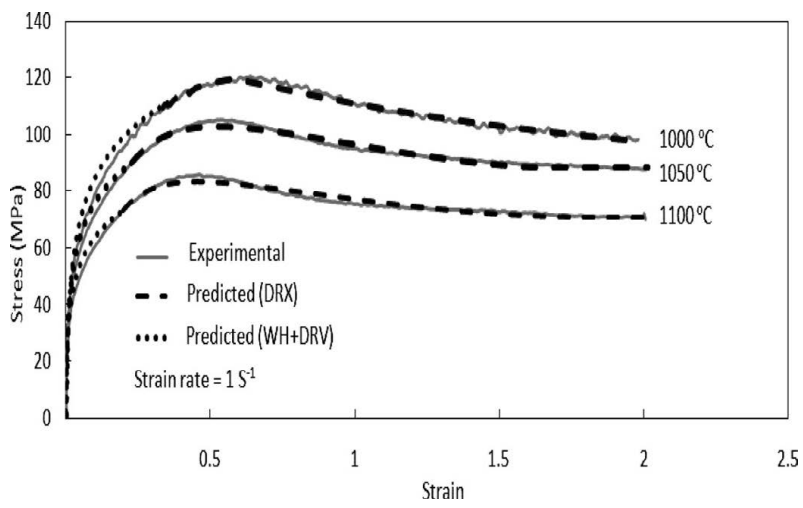

Fig. 7. Comparison of measured and predicted stress-strain curves of Ti-IF steel. 
Table 2. The variation of mean error of flow stress with true strain at temperature of $1100^{\circ} \mathrm{C}$ and strain rate of $1 \mathrm{~s}^{-1}$.

\begin{tabular}{llccc}
\hline Region & Strain & $\begin{array}{c}\text { Measured } \\
\text { Stress (MPa) }\end{array}$ & $\begin{array}{c}\text { Predicted } \\
\text { Stress (MPa) }\end{array}$ & Mean Error \% \\
\hline DRV & 0.006 & 26.07 & 26.44 & 1.32 \\
DRV & 0.168 & 69.72 & 71.1 & 1.94 \\
DRV & 0.178 & 70.89 & 71.91 & 1.41 \\
DRV & 0.194 & 72.72 & 73.04 & 0.43 \\
DRX & 0.2 & 73.77 & 74.17 & 0.52 \\
DRX & 0.23 & 76.49 & 77.28 & 1.02 \\
DRX & 0.3 & 81.52 & 80.92 & -0.27 \\
DRX & 0.4 & 84.86 & 83.15 & -2.06 \\
DRX & 0.5 & 85.31 & 83.25 & -2.47 \\
DRX & 0.6 & 83.09 & 82.35 & -0.89 \\
DRX & 0.7 & 80.26 & 81.25 & 1.21 \\
\hline
\end{tabular}

sured ones at different deformation temperature. Although, in region one, the presented model overestimates the flow stresses which can be due to the nature of logarithmic function of Eq. (11). Also the deviations of estimated flow curves in region two belong to the variations of volume fraction of DRX which is a representative of the difference between extrapolated DRV+WH and experimental DRX behavior. In order to evaluate the accuracy of the models, the mean error was calculated. The mean error of flow stress was calculated at a wide range of strains for every measurement under all deformation conditions and the maximum mean error for region one and two were calculated as $2.1 \%$ and $2.9 \%$ respectively. Table 2 shows an example of these measurements at temperature of $1100^{\circ} \mathrm{C}$ and strain rate of $1 \mathrm{~s}^{-1}$.

The results indicate that the proposed model give a good estimation of the flow stress curves. Therefore, it can be deduced that the approach to obtain a constitutive equation applicable to large strain ranges was fruitful and this presented model might have a potential to be used where more precise calculation of stress decrement due to dynamic recrystallization is important.

\section{Conclusion}

In this study, the stress-strain curve up to the critical stress was modeled on the basis of estimation of $\theta-\varepsilon$ variations, with a modification by introducing an additional temperature sensitive parameter, $k$. Also, the critical stress and strain for initiation of dynamic recrystallization (DRX) were determined using: (1) the strain hardening rate versus stress curve, (2) the constitutive equation.

The mean error of flow stress was calculated at a wide range of strains for every measurement under all deformation conditions. The results indicate that the proposed model give a good estimation of the flow stress curves. Therefore, it can be deduced that Using DRV+WH model as a complement for presented DRX model, made it possible to predict flow stress curves before and after critical strain. This analysis has been done for the stress-strain curves under hot working condition for Ti-IF steel. However, this model is not dependent on the type of material and can be extended for any condition in which a single peak dynamic recrystallization occurs.

\section{REFERENCES}

1) H. J. McQueen and N. D. Rayan: Mater. Sci. Eng. A., 322 (2002), 43.

2) M. Ueki, S. Horie and T. Nakamura: Mater. Sci. Technol., 3 (1987), 329.

3) X. He, Z. Yu and X. Lai: Computational Mater. Sci., 44 (2008), 760.

4) Y. Misaka and T. Yoshimoto: J. Jpn. Soc. Technol. Plast., 8 (1967), 414.

5) S. I. Kim, Y. Lee and S. M. Byon: J. Mater. Process. Technol., 140 (2003), 84.

6) R. Ebrahimi, S. H. Zahiri and A. Najafizadeh: J. Mater. Process. Technol., 171 (2006), 301.

7) H. Mirzadeh and A. Najafizadeh: Mater. Sci. Eng. A., 527 (2010), 1856.

8) W. L. Zhi, L. X. Song, C. Jun, Z. Hong and C. Zhen: J. Iron Steel Res. Int., 17 (2010), 51.

9) E. Shafiei and R. Ebrahimi: Mater. Sci. Eng. A., submitted paper.

10) E. I. Poliak and J. J. Jonas: ISIJ Int., 43 (2003), 684.

11) S. H. Zahiri, C. H. J. Davis and P. D. Hodgson: Scr. Mater., 52 (2005), 299.

12) A. Najafizadeh and J. J. Jonas: ISIJ Int., 46 (2006), 1679.

13) H. Mirzadeh and A. Najafizadeh: Mater. Design., 31 (2010), 1174

14) R. Ebrahimi and S. Solhjoo: Int. J. ISSI, 4 (2007), 24.

15) R. Ebrahimi: Ph. D thesis, Isfahan University of Technology, (2003), 53. 